\title{
UNIFORM ESTIMATES OF THE FUNDAMENTAL SOLUTION FOR A FAMILY OF HYPOELLIPTIC OPERATORS
}

\author{
G.CITTI, M.MANFREDINI
}

Abstract In this paper we are concerned with a family of elliptic operators represented as sum of square vector fields: $L_{\epsilon}=\sum_{i=1}^{m} X_{i}^{2}+\epsilon \Delta$, in $\mathbb{R}^{n}$ where $\Delta$ is the Laplace operator, $m<n$, and the limit operator $L=\sum_{i=1}^{m} X_{i}^{2}$ is hypoelliptic. It is well known that $L_{\epsilon}$ admits a fundamental solution $\Gamma_{\epsilon}$. Here we establish some a priori estimates uniform in $\epsilon$ of it, using a generalization of the freesing and lifting technique of Rothschild and Stein. As a consequence we deduce some a priori estimates uniform in $\epsilon$, for solutions of the approximated equation $L_{\epsilon} u=f$, and for the limit equation $L u=f$. These estimates can be used in particular while studying regularity of viscosity solutions of nonlinear equations represented in terms of vector fields.

2000 MSC: 35H10, 35A08, 43A80, 35B45.

Key words Hypoelliptic operators, Carnot groups, fundamental solution, a priori estimates

\section{INTRODUCTION}

Let $X_{1}, \ldots, X_{m}$ be smooth real vector fields on an open set $\Omega \subset \mathbb{R}^{n}$ satisfying the Hörmander condition for the hypoellipticity

$$
\operatorname{rank} \operatorname{Lie}\left(X_{1}, \ldots, X_{m}\right)(x)=n, \quad \forall x \in \Omega .
$$

It is well known that the operator

$$
L=\sum_{i=1}^{m} X_{i}^{2}
$$

is hypoelliptic, and estimates of the its fundamental solution $\Gamma$ are very well known (see [9], [20], [18], see also [22]). However, in many applications it is necessary to study elliptic regularization of this type of operators. Due to assumption (1), for every fixed point $x_{0}$ there exists a neighborhood of it (again denoted $\Omega$ ) and there exist vector fields $X_{m+1}, \ldots, X_{n} \in \operatorname{Lie}\left(X_{1}, \ldots, X_{m}\right)$ such that

$$
X_{1}, \ldots, X_{m}, X_{m+1}, \ldots, X_{n}
$$

is a basis of the tangent space at $x$ for every $x \in \Omega$. Then the operator

$$
L_{\epsilon}=\sum_{i=1}^{m} X_{i}^{2}+\epsilon \sum_{i=m+1}^{n} X_{i}^{2}
$$

is uniformly elliptic in $\Omega$. This approximation can be used to study interior regularity of viscosity solutions of nonlinear problems, when the vector fields $X_{i}$ depend

Giovanna Citti and Maria Manfredini are with Dipartimento di Matematica, Universitá di Bologna, Piazza di Porta S. Donato 5, citti@dm.unibo.it, manfredi@dm.unibo.it. The work was supported by University of Bologna: founds for selected research topics. 
on the solution: $X_{i}=X_{i}(u, \nabla u)$. We refer to [24] and [2] for nonlinear differential equation of this type, arising in complex analysis or mathematica finance. A simple example could be

$$
L u=\partial_{x}^{2} u+\left(\partial_{y}+u \partial_{z}\right)^{2} u=f, \quad u=u_{0} \text { on } \partial \Omega .
$$

This problem can not be studied directly, but, under very general assumptions on the open set $\Omega$ and the boundary datum $u_{0}$, the approximating problem:

$$
L_{\epsilon} u=f, \quad u=u_{0} \text { on } \partial \Omega,
$$

has a $C^{\infty}$ solution $u_{\epsilon}$ with gradient bounded uniformly with respect to $\epsilon$. In order to prove the existence of a classical solution of (4) it is natural to establish interior estimates uniform in $\epsilon$ for the $C^{\infty}$ solutions of (5) and then let $\epsilon$ go to 0 . As a first step in this direction we consider operators with $C^{\infty}$ coefficients, and establish uniform estimates for the fundamental solution.

The fundamental solutions of the operators $L$ and $L_{\epsilon}$ can be estimated in terms of the measure of the spheres of the the respective control distance: $d$ and $d_{\epsilon c}$. In particular, in the locally homogeneous case, there exists a constant $Q>n$, called homogeneous dimension of the space such that (see [10]):

Theoremfor every compact set $K \subset \Omega$ there exist constant $C>0$ and $C_{\epsilon}$ (dependent on $\epsilon$ ) such that for every $x, y \in K$ with $x \neq y$

$$
\begin{gathered}
|\Gamma(x, y)| \leq C d^{2-Q}(x, y), \\
\left|\Gamma_{\epsilon}(x, y)\right| \leq C_{\epsilon} d_{\epsilon c}^{2-n}(x, y) .
\end{gathered}
$$

We explicitly note that it is not possible to deduce property of the limit operator $L$ and its fundamental solution from this estimate, since the constant $C_{\epsilon}$ goes to $+\infty$ with $\epsilon$, and the exponents of the distance in (7) and (6) are different. This is due to the fact that, while studying the operator $L_{\epsilon}$, the vector fields $\epsilon X_{m+1}, \cdots, \epsilon X_{n}$ can be considered first order operators. While studying the limit operator $L$, the vector fields $X_{m+1}, \cdots, X_{n}$ only arises as commutators so that they have to be considered higher order operators, at the same point $x$.

In order to obtain uniform estimates of $\Gamma_{\epsilon}$ we will introduce here a new definition of distance $d_{\epsilon}$ in $R^{n}$, which, in the homogeneous case, provides for $L_{\epsilon}$ estimates of the form

$$
\left|\Gamma_{\epsilon}(x, y)\right| \leq C d_{\epsilon}^{2-Q}(x, y) .
$$

In other words $\Gamma_{\epsilon}$ is equivalent to the same power of $d_{\epsilon}$ as the limit fundamental solution (6) and constant $C$ is independent of $\epsilon$.

In the general case we prove the following estimate:

Theorem 1.1. For every compact set $K \subset \Omega$ and $p \in \mathbb{N}$ there exist positive constants $C, C_{p}$ independent of $\epsilon$ such that

$$
\left|\epsilon^{j} X_{i_{1}} \cdots X_{i_{p}} \Gamma_{\epsilon}(x, y)\right| \leq C_{p} \frac{d_{\epsilon}^{2-p}(x, y)}{\left|B_{\epsilon}\left(x, d_{\epsilon}(x, y)\right)\right|}, \quad i_{1}, \ldots, i_{p} \in\{1, \ldots, n\},
$$

for every $x, y \in K$ with $x \neq y$, where $j$ denotes the number of indices $i \in\{m+$ $1, \ldots, n\}$ and $B_{\epsilon}(x, r)$ denotes the ball with center $x$ and radius $r$ in the distance $d_{\epsilon}$. If $p=0$ we mean that no derivative are applied on $\Gamma$. 
Besides, for every $0<a<b$

$$
\int_{a \leq d_{\epsilon}(x, y) \leq b}\left|\epsilon^{q} X_{i_{1}} X_{i_{2}} \Gamma_{\epsilon}(x, y)\right| d y \leq C(b-a) \quad i_{1}, i_{2} \in\{1, \ldots, n\} .
$$

The main idea of the proof is a new application of the lifting method introduced in [19], together with a new definition of a distance $d_{\epsilon}$. Indeed we introduce $m$ vector fields

such that the Lie algebra generated by

$$
\tilde{X}_{n+1}, \ldots, \tilde{X}_{n+m}
$$

$$
\tilde{X}_{1}, \ldots, \tilde{X}_{m}, \tilde{X}_{n+1}+\epsilon \tilde{X}_{m+1}, \ldots, \tilde{X}_{n+m}+\epsilon \tilde{X}_{n}
$$

is free up to a suitable step $s$. These vectors eliminate any ambiguity in the order of the operators, since $\tilde{X}_{n+1}+\epsilon \tilde{X}_{m+1}, \ldots, \tilde{X}_{n+m}+\epsilon \tilde{X}_{n}$ and of first order, while $\tilde{X}_{m+1}, \ldots, \tilde{X}_{n}$ of higher order. The original operator (3) is consequently lifted to a new operator

$$
\tilde{L}_{\epsilon}=\sum_{i=1}^{m} \tilde{X}_{i}^{2}+\sum_{i=m+1}^{n}\left(\tilde{X}_{i+n-m}+\epsilon \tilde{X}_{i}\right)^{2}
$$

whose fundamental solution $\tilde{\Gamma}_{\epsilon}$, locally satisfies uniform estimates with respect to $\epsilon$. Integrating in the added variables we obtain the proof of Theorem 1.1. The distance $d_{\epsilon}$ is the projection on $\mathbb{R}^{n}$ of the control distance $\tilde{d}_{\epsilon}$ associated to $\tilde{L}_{\epsilon}$. It is not equivalent to the control distance $d_{\epsilon c}$ associated to the operator $L_{\epsilon}$ as in [18], but it has the correct homogeneity properties which allows us to prove (6). This property outline the difference from the standard Rothschild-Stein procedure, where the balls with respect to the distance $d_{\epsilon c}$ of the initial operator coincide with the projection on $\mathbb{R}^{n}$ of the balls of the lifted operator.

From Theorem 1.1 the uniform interior estimates of the solution motivating our study, immediately follow. Let $\Omega_{0} \subset \Omega$, and $W_{\epsilon, X}^{k, q}\left(\Omega_{0}\right)$ be the set of functions $f \in L^{q}\left(\Omega_{0}\right)$ such that

$$
\epsilon^{j} X_{i_{1}} \cdots X_{i_{k}} f \in L^{q}\left(\Omega_{0}\right), \quad i_{1}, \ldots, i_{k} \in\{1, \ldots, n\},
$$

with natural norm

$$
\|f\|_{W_{\epsilon, X}^{k, q}\left(\Omega_{0}\right)}=\sum_{i_{1}, \ldots, i_{k} \in\{1, \ldots, n\}} \epsilon^{j}\left\|X_{i_{1}} \ldots X_{i_{k}} f\right\|_{L^{q}\left(\Omega_{0}\right)},
$$

where $j$ denotes the number of indices $i \in\{m+1, \ldots, n\}$. We have:

Corollary 1.1. Assume that $u \in L_{\text {loc }}^{q}(\Omega)$ is a solution of

$$
L_{\epsilon} u=f \text { in } \Omega \text {, }
$$

with $f \in W_{\epsilon, X}^{k, q}(\Omega)$ and let $B(x, 2 r) \subset \subset \Omega$, where $B(x, r)$ is a sphere in the control distance associated to the vector fields $X_{1}, \ldots, X_{m}$ alone. Then there exists a constant $C$ independent of $\epsilon$ such that

$$
\|u\|_{W_{\epsilon, X}^{k+2, q}(B(x, r))} \leq C\|f\|_{W_{\epsilon, X}^{k, q}(B(x, 2 r))} .
$$

This result improves a previous result of Krylov [17], where analogous estimates of solutions of $L_{\epsilon} u=f$ are provided, but the optimal exponent in immersion is not reached. If we let $\epsilon$ go to 0 we recover the well known estimate of [19]. 


\section{Preliminaries And KNOWn Results}

In this section we review the properties of the fundamental solution of an operator of type (3), proved by [19], [8], [20]. In particular, Rothschild and Stein proved that it is always possible to assume, up to a lifting procedure, that the system of vector fields $X_{1}, \ldots, X_{m}$ is free up to order $s$ in a neighborhood of a fixed point $x_{0}$. This means that the only relations between the commutators of order less or equal to $s$ are only the ones induced by anticommutativity and Jacobi's identity. Then it is possible to complete $X_{1}, \ldots, X_{m}$ to a basis of the space, for example with the collection

$$
X_{m+1}, \ldots, X_{n}
$$

of all the commutators.

We will say that a commutator has degree $s$

$$
\operatorname{deg}(X)=s \quad \text { if } \quad X=A d\left(X_{i_{1}}, \ldots, X_{i_{s}}\right),
$$

with $i_{1}, \ldots i_{s} \in\{1, \ldots, m\}$ and we call homogeneous dimension of the space the number

$$
Q=\sum_{i=1}^{n} \operatorname{deg}\left(X_{i}\right)
$$

Via the exponential mapping a distance is naturally defined on the associated Lie group. Indeed, for every fixed point $x_{0}$ in $\mathbb{R}^{n}$ then there exist a neighborhood $V$ of $x_{0}$ and for every $x \in V$ a neighborhood $U_{x}$ of $x$ in the Lie algebra, such that for every $x \in V$ the exponential mapping

$$
u \longmapsto y=\exp \left(\sum_{i=1}^{n} u_{i} X_{i}\right)(x) .
$$

is defined in $U_{x}$. Suitable restricting $V$ and choosing $W \subset \subset V$ we can assume that for every $x \in W$ the map in (11) is defined on the same $U \subset U_{x}$ and it is a diffeomorphism from $U$ onto the image. Its inverse mapping denoted $\Theta_{x}(u)$ satisfies $U \subseteq \Theta_{x}(V)$ for every $x \in W$. Finally

$$
\Theta: W \times W \rightarrow \mathbb{R}^{n}
$$

defined by $\Theta(x, y)=\Theta_{x}(y)$ is $C^{\infty}$ on $W \times W$. For a fixed $x$, the function $\Theta_{x}$ introduces a change of variable called canonical.

Now we can define

Definition 2.1. It is called distance associated to the vector fields $X_{1}, \ldots, X_{m}$, $X_{m+1}, \ldots, X_{n}$ the function

$$
d(x, y)=\sum_{i=1}^{n}\left|u_{i}\right|^{\frac{1}{\operatorname{deg}\left(X_{i}\right)}}, \quad x, y \in W .
$$

If $\left(X_{i}\right)_{i=1, \ldots, m}$ are nilpotent and free the exponential map is a global isomorphism between the free and nilpotent Lie algebra $g(m, s)$, and its associated Lie group $G(m, s)$. Hence we can assume that it is simply the identity. Since on the algebra a natural dilation is defined, via the exponential map a dilation is also induced on $G(m, s)$, which becomes an homogeneous Carnot group with homogeneous dimension $Q$. In this case the fundamental solution of the sublaplacian operator (2) is represented as

$$
\Gamma(x, y)=k_{0}(\Theta(x, y))
$$


where $k_{0}$ is an homogeneous kernel of order $Q-2$. By the homogeneity properties, it also follows that, for every $p \in \mathbb{N}$ there exists a positive constant $c_{p}$ such that for every $i_{1} \ldots, i_{p} \in\{1, \ldots, m\}$, one has

$$
\left|X_{i_{1}} \cdots X_{i_{p}} \Gamma(x, y)\right| \leq c_{p} d^{2-Q-p}(x, y) .
$$

In case the vector fields $X_{i}$ are free only up to a step $s$, then the exponential map is only a local diffeomorphism between the Lie algebra and the group. However, via the canonical coordinates, Rothschild and Stein proved that it is again possible to reduce to the homogeneous case:

Theorem 2.1. (Theorem 5 in [19]) In the u-coordinates given by $\Theta_{x}$ and in the neighborhoods $U, W$ defined in (11) we can write

$$
\tilde{X}_{i}=Y_{i}+R_{i}^{x}, \quad \forall x \in W
$$

on $U$, with $Y_{i}$ generators of the free Lie algebra with $m$ generators and step $s$, and $R_{i}^{x}$ a vector field of degree $\leq 0$ depending smoothly on $x \in W$.

In this case a parametrix of the fundamental solution of the operator $L$ in (2) is given in terms of suitable homogeneous kernels.

Theorem 2.2. (Theorem 2 in [20]) Let $a \in C_{0}^{\infty}(W)$ and $p \in \mathbb{N}$. There exist $k^{i}$ homogeneous kernels on the group $G(m, s)$, functions $a_{i}, b_{i} \in C_{0}^{\infty}(W), T_{i}(x, y)$ depending on the derivatives of $\Theta$ of order $p, E_{p} \in C^{\infty}(W \times W)$ such that the function

$$
K_{p}(x, y)=\sum_{i=1}^{d} a_{i}(x) k^{i}(\Theta(x, y)) T_{i}(x, y) b_{i}(y)+E_{p}(x, y)
$$

is a parametrix of the fundamental solution. It satisfies

$$
L^{x}\left(K_{p}(x, y)\right)=a(x) \delta_{y}(x)+H_{p}(x, y)
$$

where $H_{p} \in C_{0}^{p}(W \times W), \delta_{y}$ is the Dirac distribution at $y$, and $L^{x}$ means that the differentiation is in the $x$-variable.

From this theorem Sánchez-Calle deduces the well known estimates on the fundamental solution:

Theorem 2.3. For every compact set $K \subset \Omega$ and integer $p \geq 0$ there exists positive constant $C_{p}$ such that for every $x, y \in K$ with $x \neq y$

$$
\left|X_{i_{1}} \cdots X_{i_{p}} \Gamma(x, y)\right| \leq C_{p} \frac{d^{2-p}(x, y)}{\left|B_{d}(x, d(x, y))\right|}, \quad i_{1}, \ldots, i_{p} \in\{1, \ldots, m\} .
$$

\section{UNIFORM ESTIMATES}

In this section we proof uniform estimates for the fundamental solution of $L_{\epsilon}$ in (3), both in the homogeneous and in the general situation. 
3.1. The homogeneous case. Let $X_{1}, \ldots, X_{m}$ be a family of homogeneous real vector fields. Up to a change of variables we can assume that $X_{i}$ agrees at origin with $\partial_{x_{i}}$, and, up to a lifting, that their generated Lie algebra is the free and nilpotent Lie algebra $g(m, s)$. We complete the collection $X_{i}, \ldots, X_{m}$ to a basis of $R^{n}$

$$
X_{1}, \ldots, X_{m}, X_{m+1}, \ldots, X_{n}
$$

A possible choice of $X_{i}, \ldots, X_{m}$ is the family of all the commutators of step $\leq s$.

The generic point of the space is denoted $x \in R^{n}$. In order to prove Theorem 1.1 we perform a modification of the lifting procedure. We choose $n-m$ new variables of the space and $n-m$ new vector fields $X_{n+1}, \ldots, X_{2 n-m}$, depending on these new variables, free and nilpotent of step $s$. By Theorem 4 in [19], there exist families of vector fields $\tilde{X}_{1}, \ldots, \tilde{X}_{m}$ lifting $X_{1}, \ldots, X_{m}$ and $\tilde{X}_{n+1}, \ldots, \tilde{X}_{2 n-m}$ lifting $X_{n+1}, \ldots, X_{2 n-m}$, such that

$$
\tilde{X}_{1}, \ldots, \tilde{X}_{m}, \tilde{X}_{n+1}, \ldots, \tilde{X}_{2 n-m}
$$

are generators of the free Lie algebra $g(n, s)$.

In this lifting procedure the vector fields $X_{m+1}, \ldots, X_{n}$ will be lifted to suitable vector fields, denoted

$$
\tilde{X}_{m+1}, \ldots, \tilde{X}_{n}
$$

The dimension of the vector space will now be denoted $N$.

The generic point will be $\tilde{x}=(x, \hat{x})$, where $x \in R^{n}$ denotes the initial variables, $\hat{x} \in R^{N-n}$ the added ones. In the initial variables we have canonical coordinates on $\mathbb{R}^{n}$ around any fixed point $x, \Theta_{x}(y)=u$ and the distance $d$ associated to $X_{1}, \ldots, X_{n}$ defined as in Definition 2.1. In the lifted space $\mathbb{R}^{N}$ we can complete the family $\tilde{X}_{1}, \ldots, \tilde{X}_{n+m}$ previously defined to a basis of the Lie algebra. The resulting basis will consequently be denoted:

$$
\tilde{X}_{1}, \ldots, \tilde{X}_{m}, \tilde{X}_{m+1}, \ldots, \tilde{X}_{n}, \tilde{X}_{n+1}, \ldots, \tilde{X}_{2 n-m}, \tilde{X}_{2 n-m+1}, \ldots, \tilde{X}_{N} .
$$

We will denote $\Theta_{\tilde{x}}(\tilde{y})=\tilde{u}$ the canonical coordinates with respect to this basis, and $\tilde{d}$ the associated distance, according to Definition 2.1 and $\tilde{Q}$ the homogeneous dimension according to (10).

Also the family

$$
\tilde{X}_{\epsilon, i}=\tilde{X}_{i}, i=1, \ldots, m, \quad \tilde{X}_{\epsilon, i}=\tilde{X}_{i}+\epsilon \tilde{X}_{i-n+m}, i=n+1, \ldots, 2 n-m
$$

is a family of generators for the Lie algebra $g(n, s)$. Let $\psi_{\epsilon}$ be a Lie algebra isomorphism defined on the generators as

$$
\psi_{\epsilon}\left(\tilde{X}_{i}\right)=\tilde{X}_{\epsilon, i}, \quad i=1, \ldots, m, \quad \text { and } \quad i=n+1, \ldots, 2 n-m .
$$

Clearly $\psi_{\epsilon}$ can be extended on the whole algebra via the bracket, so that

$$
\tilde{X}_{\epsilon, i}=\psi_{\epsilon}\left(\tilde{X}_{i}\right)=\tilde{X}_{i}+\sum_{j=1}^{N} a_{i j} \tilde{X}_{j}, \quad \forall i=1, \ldots, N
$$

where

$$
a_{i j} \leq \epsilon \quad \forall i, j \in\{1, \ldots, N\} .
$$

Then one easily checks that the jacobian determinant of $\psi_{\epsilon}$ is 1 . We denote by $\tilde{d}_{\epsilon}$ the distance associated to $\tilde{X}_{\epsilon, 1}, \ldots, \tilde{X}_{\epsilon, N}$ according to Definition 2.1. 

$\mathbb{R}^{N}$

The function $\psi_{\epsilon}$ induces, via the mapping $\Theta$, also a change of variables on group

$$
\Phi_{\epsilon}: \mathbb{R}^{N} \rightarrow \mathbb{R}^{N}, \quad \Phi_{\epsilon}=\operatorname{Exp} \circ \phi_{\epsilon} \circ \Theta_{0} .
$$

Obviously also the jacobian determinant of $\Phi_{\epsilon}$ is independent of $\epsilon$ at every point, besides

$$
\tilde{d}_{\epsilon}(\tilde{x}, \tilde{y})=\sum_{i=1}^{N}\left|\Phi_{\epsilon}(\tilde{u})_{i}\right|^{\frac{1}{\operatorname{deg}\left(\psi_{\epsilon}\left(\tilde{X}_{i}\right)\right)}}=\tilde{d}\left(\Phi_{\epsilon}(\tilde{x}), \Phi_{\epsilon}(\tilde{y})\right) .
$$

We can now define two lifted operators:

$$
\tilde{L}=\sum_{i=1}^{m} \tilde{X}_{i}^{2}+\sum_{i=n+1}^{2 n-m} \tilde{X}_{i}^{2}
$$

and

$$
\tilde{L}_{\epsilon}=\sum_{i=1}^{m} \tilde{X}_{\epsilon, i}^{2}+\sum_{i=n+1}^{2 n-m} \tilde{X}_{\epsilon, i}^{2}=\sum_{i=1}^{m} \tilde{X}_{\epsilon, i}^{2}+\sum_{i=m+1}^{n}\left(\tilde{X}_{i+n-m}+\epsilon \tilde{X}_{i}\right)^{2} .
$$

The estimate of $\Gamma_{\epsilon}$ independent of $\epsilon$ will be obtained by comparison of these two lifted operator. Indeed, the first is independent of $\epsilon$ and has exactly the same structure of one second one, and the change of variables $\Phi_{\epsilon}$ which change the first operator in the second one has jacobian determinant independent of $\epsilon$

Note that the balls $B_{d}(x, r)$ in the distance $d$ are projection on $\mathbb{R}^{n}$ of the balls $B_{\tilde{d}}((x, 0), r)$ in the distance $\tilde{d}$. Indeed, we can define

$$
\pi_{1}: \mathbb{R}^{N} \longrightarrow \mathbb{R}^{n}, \quad \pi_{1}(x, \widehat{x})=x
$$

and apply the following result

Lemma 3.1. (Lemma 3.1 in [18])

$$
\pi_{1}: B_{\tilde{d}}((x, 0), r) \longrightarrow B_{d}(x, r),
$$

and the map is onto.

This is not the case of the balls $B_{d_{\epsilon c}}(x, r)$ in the control distance $d_{\epsilon c}$ associated to $L_{\epsilon}$, which are not the projection of the balls $\tilde{B}_{\epsilon}((x, 0), r)$ associated to the operator $\tilde{L}_{\epsilon}$.

According to Lemma 3.1 we define a new distance $d_{\epsilon}$ on $\mathbb{R}^{n}$, which as we ..? is different from the ones introduced in [18] and [20], and which will be used to estimate the fundamental solution for the operator $L_{\epsilon}$.

We will denote

$$
B_{\epsilon}(x, r)=\pi_{1}\left(\tilde{B}_{\epsilon}((x, 0), r)\right)
$$

where $\tilde{B}_{\epsilon}$ is the ball associated to $\tilde{d}_{\epsilon}$.

Lemma 3.2. The family of balls $B_{\epsilon}$ defines a quasi-distance $d_{\epsilon}$ on $\mathbb{R}^{n}$.

See [25] page 11.

If $\tilde{\Gamma}$ is the fundamental solution for $\tilde{L}$, and $\tilde{\Gamma}_{\epsilon}$ the fundamental solution for the regularized operator $\tilde{L}_{\epsilon}$, then

$$
\tilde{\Gamma}_{\epsilon}(\tilde{x}, \tilde{y})=\tilde{\Gamma}\left(\Phi_{\epsilon}(\tilde{x}), \Phi_{\epsilon}(\tilde{y})\right) .
$$


If $p \in \mathbb{N}$ then there exists a positive constant $C_{p}$ such that for every $i_{1} \ldots, i_{p} \in$ $\{1, \ldots, m\}$, one has

$$
\left|\tilde{X}_{\epsilon, i_{1}} \cdots \tilde{X}_{\epsilon, i_{p}} \tilde{\Gamma}_{\epsilon}(\tilde{x}, \tilde{y})\right| \leq C_{p}\left(\tilde{d}\left(\Phi_{\epsilon}(\tilde{x}), \Phi_{\epsilon}(\tilde{x})\right)^{2-Q-p} .\right.
$$

The constant $C_{p}$ is independent of $\epsilon$. We are interested in a similar estimates for the fundamental solution $\Gamma_{\epsilon}$.

Lemma 3.3. For every compact set $K \subset \mathbb{R}^{n}$ there exist positive constants $C_{1}, C_{2}$ independent of $\epsilon$ such that if $\tilde{B}_{\epsilon}((x, 0), r)$ denotes the ball in the metric $\tilde{d}_{\epsilon}$, and $\chi_{\tilde{B}_{\epsilon}((x, 0), r)}$ is the characteristic function of the ball $\tilde{B}_{\epsilon}((x, 0), r)$, then for every $x \in K$ and $r>0$

$$
C_{1} r^{\tilde{Q}-Q} \leq \int \chi_{\tilde{B}_{\epsilon}((x, 0), r)} d \hat{y} \leq C_{2} r^{\tilde{Q}-Q} .
$$

Note that the integration is performed only in the added variables $\hat{y}$.

Proof

$$
\int \chi_{\tilde{B}_{\epsilon}((x, 0), r)} d \hat{y}=\left|\left\{\hat{y}: \exists y \in \mathbb{R}^{n}:(y, \hat{y}) \in \tilde{B}_{\epsilon}((x, 0))\right\}\right|=
$$

by definition of $B_{\epsilon}$

$$
\begin{gathered}
=\left|\bigcup_{y \in B_{\epsilon}(x, r)}\left\{\hat{y}: \tilde{d}_{\epsilon}((y, \hat{y}),(x, 0))<r\right\}\right|= \\
=\left|\bigcup_{y \in B_{\epsilon}(x, r)}\left\{\hat{y}:(y, \hat{y})=\exp \left(\sum_{i=1}^{n} u_{\epsilon, i} \tilde{X}_{\epsilon, i}+\sum_{i=n+1}^{N} \hat{u}_{\epsilon, i} \tilde{X}_{\epsilon, i}\right)(x, 0),\left|\tilde{u}_{\epsilon, i}\right|^{\frac{1}{d e g\left(\tilde{X}_{\epsilon, i}\right)}}<r\right\}\right|,
\end{gathered}
$$

recall that $\tilde{u}=(u, \hat{u})$. By Lemma 3.2 in [18] there exist constants $\eta_{1}, \eta_{2}, C>0$, $0<\eta_{2}<\eta_{1}<1$, only depending on the Campbell-Hausdorff formula, such that $\forall y \in B_{\epsilon}(x, r), \forall \hat{u}_{\epsilon}$, such that $\left|\hat{u}_{\epsilon, j}\right| \leq \eta_{2} r^{\operatorname{deg}\left(\tilde{X}_{\epsilon, j}\right)}$ for every $j=n+1, \ldots, N$, there exists unique $u_{\epsilon}$, and $\hat{y}$ such that $\left|u_{\epsilon, j}\right| \leq \eta_{1} r^{\operatorname{deg}\left(X_{\epsilon, j}\right)}$ for every $j=1, \ldots, n$, and

$$
(y, \hat{y})=\exp \left(\sum_{i=1}^{n} u_{\epsilon, i} \tilde{X}_{\epsilon, i}+\sum_{i=n+1}^{N} \hat{u}_{\epsilon, i} \tilde{X}_{\epsilon, i}\right)(x, 0) .
$$

Besides the map

$$
\hat{u} \mapsto \hat{y}
$$

is a diffeomorphism, with jacobian determinant only depending on the constant $C$. Then

$$
\int \chi_{\tilde{B}_{\epsilon}((x, 0), r)} d \hat{y} \leq\left|\left\{\hat{u}_{\epsilon}:\left|\hat{u}_{\epsilon, j}\right| \leq \eta_{2} r^{\operatorname{deg}\left(\tilde{X}_{\epsilon, j}\right)}, \forall j=n+1, \ldots, N\right\}\right|=
$$

(since the restriction of $\psi_{\epsilon}$ to the subspace generated by the new variables $\hat{u}$ has still determinant 1)

$$
=\left|\left\{\hat{u}_{:}\left|\hat{u}_{j}\right| \leq \eta_{2} r^{\operatorname{deg}\left(\tilde{X}_{j}\right)}, \forall j=n+1, \ldots, N\right\}\right|=C r^{\tilde{Q}-Q}
$$

where the last inequality does not depend on $\epsilon$ any more.

The remaining inequality is obvious because of

$$
\left|\bigcup_{y \in B_{\epsilon}(x, r)}\left\{\hat{y}:(y, \hat{y})=\exp \left(\sum_{i=1}^{n} u_{\epsilon, i} \tilde{X}_{\epsilon, i}+\sum_{i=n+1}^{N} \hat{u}_{\epsilon, i} \tilde{X}_{\epsilon, i}\right)(x, 0),\left|\tilde{u}_{\epsilon, i}\right| \leq \eta_{1} r^{\operatorname{deg}\left(\tilde{X}_{\epsilon, i}\right)}\right\}\right|
$$




$$
\geq\left|\left\{\hat{u}_{\epsilon}:\left|\hat{u}_{\epsilon, j}\right| \leq \eta_{2} r^{\operatorname{deg}\left(\tilde{X}_{\epsilon, j}\right)}, \forall j=n+1, \ldots, N\right\}\right| .
$$

Remark 3.1. We have

$$
c_{1} r^{Q} \leq\left|B_{\epsilon}(x, r)\right| \leq c_{2} r^{Q},
$$

with $c_{1}, c_{2}$ positive constant independent of $\epsilon$. Indeed, by Lemma 3.3

$$
\begin{gathered}
\left|B_{\epsilon}(x, r)\right| \leq \int_{B_{\epsilon}(x, r)} \frac{c}{r^{\tilde{Q}-Q}} \int \chi_{\tilde{B}_{\epsilon}((x, 0), r)} d \hat{y} d y \leq \\
\leq \frac{c}{r^{\tilde{Q}-Q}} \int_{\pi_{1}\left(\tilde{B}_{\epsilon}((x, 0), r)\right)} \int \chi_{\tilde{B}_{\epsilon}((x, 0), r)} d \hat{y} d y=\frac{c}{r^{\tilde{Q}-Q}} \int_{\tilde{B}((x, 0), r)} d \hat{y} d y=c_{2} r^{Q} .
\end{gathered}
$$

We can now conclude in a standard way the proof that $\Gamma_{\epsilon}$ is a regular kernel:

Proof of Theorem 1.1 for homogeneous vector fields. The proof is a consequence of Remark 3.1, which is uniform in $\epsilon$. For reader convenience we provide here estimate (8) with $p=0$. It is known (see for example [19], pag 302) that a local parametrix for $\Gamma_{\epsilon}$ is provided by

$$
\int \tilde{\Gamma}_{\epsilon}((x, 0),(y, \hat{y})) b(\hat{y}) d \hat{y}
$$

where $b$ is any function of class $C^{\infty}$ with compact support. On the other side

$$
\begin{gathered}
\int \tilde{\Gamma}_{\epsilon}((x, 0),(y, \hat{y})) b(\hat{y}) d \hat{y} \leq \sum_{j} \int_{\eta 2^{-j} \leq \tilde{d}_{\epsilon}((x, 0),(y, \hat{y})) \leq \eta 2^{-j+1}} \tilde{\Gamma}_{\epsilon}((x, 0),(y, \hat{y})) b(\hat{y}) d \hat{y} \leq \\
\quad \leq c \sum_{j} \int_{\eta 2^{-j} \leq \tilde{d}_{\epsilon}((x, 0),(y, \hat{y})) \leq \eta 2^{-j+1}}\left(\tilde{d}_{\epsilon}((x, 0),(y, \hat{y}))\right)^{-\tilde{Q}+2} d \hat{y} \leq c d_{\epsilon}^{2-Q}(x, y),
\end{gathered}
$$

since in each term of the sum $d_{\epsilon}(x, y) \leq \tilde{d}_{\epsilon}((x, 0),(y, \hat{y})) \leq \eta 2^{-j+1}$.

Also note that we have the following local inclusions, which ensure that local estimates uniform in $\epsilon$ with respect to $d_{\epsilon}$ are local estimates uniform in $\epsilon$ with respect to the distance $d$ :

Lemma 3.4. For every compact set $K \subset \mathbb{R}^{n}$ there exists a positive constant $C$ independent of $\epsilon$ such that for every $x \in K$

$$
B_{\epsilon}(x, r) \subset B\left(x, C\left(r+(\epsilon r)^{\frac{1}{s}}\right)\right)
$$

and

$$
B(x, r) \subset B_{\epsilon}\left(x, C\left(r+(\epsilon r)^{\frac{1}{s}}\right)\right),
$$

where $s$ is the step of Lie algebra.

Proof Let us consider a point $y \in B_{\epsilon}(x, r)$. Then there exists $\hat{y} \in \mathbb{R}^{N-n}$ such that

$$
(y, \hat{y})=\exp \left(\sum_{i=1}^{N} \tilde{u}_{\epsilon, i} \tilde{X}_{\epsilon, i}\right)(x, 0) \text { and }\left|\tilde{u}_{\epsilon, i}\right|^{\frac{1}{\operatorname{deg}\left(\tilde{X}_{\epsilon, i}\right)}}<r .
$$

By (15) we can write

$$
(y, \hat{y})=\exp \left(\sum_{i=1}^{N} \tilde{u}_{\epsilon, i}\left(\tilde{X}_{i}+\sum_{j=1}^{N} a_{i j} \tilde{X}_{j}\right)\right)(x, 0)=\exp \left(\sum_{j=1}^{N} \sum_{i=1}^{N} \tilde{u}_{\epsilon, i}\left(\delta_{i j}+a_{i j}\right) \tilde{X}_{j}\right)(x, 0) .
$$


Now

$$
\left|\tilde{u}_{\epsilon, i}\left(\delta_{i j}+a_{i j}\right)\right|^{\frac{1}{\operatorname{deg}\left(\tilde{X}_{j}\right)}} \leq
$$

by (16) and the fact that $\left.\operatorname{deg} \tilde{(} X_{j}\right) \leq s$

$$
\leq C\left|\tilde{u}_{\epsilon, j}\right|^{\frac{1}{\operatorname{deg}\left(\tilde{X}_{j}\right)}}+\left|\epsilon \tilde{u}_{\epsilon, i}\right|^{\frac{1}{s}} \leq C\left(r+(\epsilon r)^{\frac{1}{s}}\right),
$$

by (19). It follows that $y \in B\left(x, C\left(r+(\epsilon r)^{\frac{1}{s}}\right)\right)$.

\section{Proof of Corollary $\mathbf{1 . 1}$ for homogeneous vector fields}

$$
L_{\epsilon} u=f \text { in } \Omega \text {, }
$$

with $f \in W_{\epsilon, X}^{k, q}(\Omega)$ and let $B(x, 8 r) \subset \subset \Omega$. Then $B_{\epsilon}(x, 4 r) \subset \subset \Omega$ for every $\epsilon$. From the preceding Theorem 1.1 and standard properties of singular integrals it immediately follows that

$\|u\|_{W_{\epsilon, X}^{k+2, q}(B(x, r))} \leq\|u\|_{W_{\epsilon, X}^{k+2, q}\left(B_{\epsilon}(x, 2 r)\right)} \leq C\|f\|_{W_{\epsilon, X}^{k, q}\left(B_{\epsilon}(x, 4 r)\right)} \leq C\|f\|_{W_{\epsilon, X}^{k, q}(B(x, 8 r))}$.

3.2. The general case. Let $X_{1}, \ldots, X_{m}$ be a family of smooth real vector fields on $\mathbb{R}^{n}$ satisfying the Hörmander condition (1). Then the preceding construction has to be slightly modified. Indeed, up to a lifting we can assume that the given vector fields are free at step $s$ in a neighborhood $U$ of $x_{0}$. Let us denote

$$
X_{m+1}, \ldots, X_{n}
$$

a completion of the basis of the space. The exponential map is now only a local diffeomorphis between the Lie algebra and the group. For a fixed $x_{0}$, as in Definition 2.1 there exist neighborhood $U$ of 0 and neighborhoods $V, W$ of $x_{0}$ on which the exponential mapping is a diffeomorphism. We will denote

$$
\Theta_{x}(y)=u
$$

the canonical coordinates induced by $X_{1}, \ldots, X_{n}$.

In the fixed neighborhood of $x_{0}$ the vectors $\left(X_{i}\right)_{i=1, \ldots, m}$ can be represented in local coordinates in terms of homogeneous vector fields, $\left(Y_{i}\right)_{i=1, \ldots, m}$ as recalled in Theorem 2.1.

Let us define frozen vector fields also for the remaining vectors

$$
\epsilon X_{m+1}, \ldots, \epsilon X_{n} \text {. }
$$

We explicitly note that if

$$
X_{k}=\operatorname{Ad}\left(X_{i_{1}}, \ldots, X_{i_{p}}\right),
$$

we can not simply take as frozen vector of $\epsilon X_{k}$ the vector

$$
\epsilon A d\left(Y_{i_{1}}, \ldots, Y_{i_{p}}\right),
$$

Indeed, applying iteratively Theorem 2.1 we get

$$
\epsilon X_{k}=\epsilon A d\left(X_{i_{1}}, \ldots, X_{i_{p}}\right)=\epsilon A d\left(Y_{i_{1}}, \ldots, Y_{i_{p}}\right)+R_{k},
$$

where $R_{k}$ has degree less or equal to $k-1$. On the other hand while studying the operator $L_{\epsilon}$ the vectors $\epsilon A d\left(X_{i_{1}}, \ldots, X_{i_{p}}\right)$ has to be considered of degree 1 , and we need an approximation with homogeneous vectors, and rest of degree $\leq 0$. 
Theorem 3.1. Let $W$ be the fixed neighborhood of $x_{0}$ in $\mathbb{R}^{n}$. There exist vectors $\left(Y_{i}\right)_{i=m+1, \ldots, n}$ which complete $Y_{1}, \ldots, Y_{m}$ to a basis, and such that in the $u-$ coordinates we have

$$
\epsilon X_{i}=\epsilon Y_{i}+R_{i}^{x} \quad i=m+1, \ldots, n
$$

on $U$, and $R_{i}^{x}$ a vector field of degree $\leq 0$ depending smoothly on $x \in W$. In general $Y_{m+1}, \ldots, Y_{n}$ will not be the enumeration of all the commutator of higher degree, but could also contains linear combination of them.

Proof. We perform the proof by induction on the degree of the vectors $X_{i}$. Let us first assume that $\operatorname{deg}\left(X_{i}\right)=2$. This means that there exist indices $i_{1}, i_{2}$ such that $X_{i}=\left[X_{i_{1}}, X_{i_{2}}\right]$. By Theorem 2.1 there exist vector fields $Y_{i_{1}}$ and $Y_{i_{2}}$ such that

$$
X_{i_{1}}=Y_{i_{1}}+R_{i_{1}}, \quad X_{i_{2}}=Y_{i_{1}}+R_{i_{1}}
$$

with $\operatorname{deg}\left(R_{i_{1}}\right) \leq 0$. Then

$X_{i}=\left[X_{i_{1}}, X_{i_{2}}\right]=\left[Y_{i_{1}}+R_{i_{1}}, Y_{i_{2}}+R_{i_{2}}\right]=\left[Y_{i_{1}}, Y_{i_{2}}\right]+\left[Y_{i_{1}}, R_{i_{2}}\right]+\left[R_{i_{1}}, Y_{i_{2}}\right]+\left[R_{i_{1}}, R_{i_{2}}\right]$.

By Theorem 2.1 there exist vectors

$$
\left[Y_{i_{1}}, R_{i_{2}}\right]=E_{12}+R_{12},\left[R_{i_{1}}, Y_{i_{2}}\right]=E_{21}+R_{21}
$$

with $\operatorname{deg}\left(R_{12}\right) \leq 0, \operatorname{deg}\left(R_{21}\right) \leq 0$. Then the vector $\left[Y_{i_{1}}, Y_{i_{2}}\right]+E_{12}+E_{21}$ provides the required approximation of $X_{i}$. The induction step is similar and we omit it.

We can now consider the frozen operator

$$
L_{\epsilon, Y}=\sum_{i=1}^{m} Y_{i}^{2}+\epsilon \sum_{i=m+1}^{n} Y_{i}^{2} .
$$

By the results in subsection 3.1 there exist a natural distance $d_{\epsilon, Y}$ associated to $L_{\epsilon, Y}$ and a fundamental solution $\Gamma_{\epsilon, Y}$ which is a regular kernel in the sense of the following definition:

Definition 3.1. We will say that $k_{\epsilon}$ is an regular kernel of type $\lambda$ with respect to the vectors $X_{1}, \ldots, X_{m}, \epsilon X_{m+1}, \ldots, \epsilon X_{n}$, and the distance $d_{\epsilon}$ in an open set $W$ and we will denote $k_{\epsilon} \in F_{\lambda}\left(X, d_{\epsilon}, W\right)$ if for every $x, y \in W$ with $x \neq y$

$$
\begin{gathered}
\left|k_{\epsilon}(x, y)\right| \leq C_{0} \frac{d_{\epsilon}^{\lambda}(x, y)}{\left|B_{\epsilon}\left(x, d_{\epsilon}(x, y)\right)\right|}, \\
\left|\epsilon^{j} X_{i_{1}} \cdots X_{i_{p}} k_{\epsilon}(x, y)\right| \leq C_{p} \frac{d_{\epsilon}^{\lambda-p}(x, y)}{\left|B_{\epsilon}\left(x, d_{\epsilon}(x, y)\right)\right|}, \quad i_{1}, \ldots, i_{p} \in\{1, \ldots, n\},
\end{gathered}
$$

where $j$ is the number of indices greater to $m$. If $\lambda=0$ we also require that

$$
\int_{a \leq d_{\epsilon}(x, y) \leq b}\left|k_{\epsilon}(x, y)\right| \operatorname{det} J \Theta_{x}(y) \mid d y \leq C_{*}(b-a) .
$$

We now follow the same construction as in [20], in order to define a parametrix for the fundamental solution of $L_{\epsilon}$, in terms of the fundamental solution of $L_{\epsilon, Y}$. The main difference from the proof in [20] are due to the fact that our model operator $L_{\epsilon, Y}$ is not homogeneous, and that we need to study the dependence on $\epsilon$.

We first introduce a distance $d_{\epsilon}$ as follows:

$$
d_{\epsilon}(x, y)=d_{\epsilon, Y}(0, \Theta(x, y))
$$

and denote $B_{\epsilon}(x, r)$ the associated spheres. 
With this definition it is clear that there is a natural relation between kernels of type $F_{\lambda}\left(Y_{\epsilon}, d_{\epsilon, Y}, U\right)$, and $F_{\lambda}\left(X_{\epsilon}, d_{\epsilon}, W\right)$. Indeed

Proposition 3.1. If $k(x, y)$ is a kernel of class $F_{\lambda}\left(Y_{\epsilon}, d_{\epsilon, Y}, U\right)$, then $k(0, \Theta(x, y))$ is a kernel of class $F_{\lambda}\left(X_{\epsilon}, d_{\epsilon}, W\right)$, which satisfies inequalities (20), (21), (22), with the same constants as $k$.

Note that these kernels have the following properties:

Proposition 3.2. If $k_{0}$ is a kernel of class $F_{2}\left(Y_{\epsilon}, d_{\epsilon, Y}, U\right)$, which satisfies conditions (20), (21) with constants $C_{0}, C_{p}$, and $k$ is a kernel of class $F_{\lambda}\left(Y_{\epsilon}, d_{\epsilon, Y}, U\right)$, $\lambda \geq 1$, which satisfies conditions (20), (21) with constants $\tilde{C}_{0}, \tilde{C}_{p}$, then there exist constants $D_{0}, D_{p}$, only dependent on $C_{i}$ and $\tilde{C}_{i}$ such that the kernel

$$
k_{1}(x, y)=\int k(x, y) k_{0}(y, z) d y
$$

is of class $F_{\lambda+2}\left(Y_{\epsilon}, d_{\epsilon, Y}, U\right)$, and satisfies (20), (21) with constants $D_{0}, D_{p}$.

Theorem 3.2. Let $a \in C_{0}^{\infty}(W), p \in \mathbb{N}$. There exist kernels $K_{\epsilon, p} \in F_{2}\left(X_{\epsilon}, d_{\epsilon}, W\right)$ and $H_{\epsilon, p} \in F_{p}\left(X_{\epsilon}, d_{\epsilon}, W\right)$, satisfying (20), (21) with constants independent of $\epsilon$ such that

$$
L_{\epsilon}^{x}\left(K_{\epsilon, p}(x, y)\right)=a(x) \delta_{y}(x)+H_{\epsilon, p}(x, y)
$$

with $\delta_{y}$ the Dirac distribution at $y$, and where $L_{\epsilon}^{x}$ means that the differentiation is in the $x$-variable.

Proof The proof is similar to Theorem 2 in [20]. We set

$$
K_{\epsilon, 0}(x, y)=a(x) \Gamma_{\epsilon, Y}(0, \Theta(x, y)) b(y)
$$

then by Theorem 3.1

$$
L_{\epsilon}^{x}\left(K_{\epsilon, 0}(x, y)\right)=a(x) \delta_{y}(x)+H_{\epsilon, 1}(x, y)
$$

with $H_{\epsilon, 1} \in F_{1}\left(X_{\epsilon}, d_{\epsilon}, W\right)$. We obtain the function $H_{\epsilon, 1}$ while differentiating twice $K_{\epsilon, 0}(x, y)$, and applying the derivatives to the functions $a$, or while we apply one derivative on $a$, and a derivative on $\Gamma_{\epsilon, Y}(0, \Theta(x, y))$. In both cases the kernel satisfies conditions (20), (21) uniformly in $\epsilon$, by Proposition 3.1, and the proof of Theorem 1.1 for homogeneous vector fields. Assume $K_{\epsilon, p} \in F_{2}\left(X_{\epsilon}, d_{\epsilon}, W\right), H_{\epsilon, p} \in$ $F_{p}\left(X_{\epsilon}, d_{\epsilon}, W\right)$ satisfies conditions $(20),(21)$ uniformly in $\epsilon$. Then

$$
L_{\epsilon}^{x}\left(K_{\epsilon, p}(x, y)\right)=a(x) \delta_{y}(x)+H_{\epsilon, p}(x, y)
$$

and $H_{\epsilon, p}(x, y)=c(x) k_{\epsilon, Y}(0, \Theta(x, y)) b(y)$. Define

$$
K_{\epsilon, p+1}=K_{\epsilon, p}-c(x) k_{\epsilon, Y} * \Gamma_{\epsilon, Y}(0, \Theta(x, y)) b(y) .
$$

Then $K_{\epsilon, p} \in F_{p}\left(X_{\epsilon}, d_{\epsilon}, W\right)$, and satisfies conditions (20), (21) uniformly in $\epsilon$, by Proposition 3.2. Besides, $L_{\epsilon}^{x}\left(K_{\epsilon, p+1}(x, y)\right)=a(x) \delta_{y}(x)+H_{\epsilon, p+1}(x, y)$ with $H_{\epsilon, p+1} \in$ $F_{p+1}\left(X_{\epsilon}, d_{\epsilon}, W\right)$.

Proof of Theorem 1.1 Let us fix $x \in \Omega$ and $p \in \mathbb{N}$, and call the function

$$
\begin{aligned}
F_{\epsilon}(u, v)=\Gamma_{\epsilon} & \left(\exp \left(\sum_{i=1}^{n} u_{i} X_{i}\right)(x), \exp \left(\sum_{i=1}^{n} v_{i} X_{i}\right)(x)\right)- \\
& -K_{\epsilon, p}\left(\exp \left(\sum_{i=1}^{n} u_{i} X_{i}\right)(x), \exp \left(\sum_{i=1}^{n} v_{i} X_{i}\right)(x)\right)
\end{aligned}
$$


where $K_{\epsilon, p}$ is defined in Theorem 3.2. By Theorems 3.2 and 3.1 there exists a function $H_{\epsilon, p} \in F_{p}\left(X_{\epsilon}, d_{\epsilon}, W\right)$ with defining constants, bounded uniformly in $\epsilon$, such that

$$
L_{\epsilon, Y} F_{\epsilon}(u, v)=H_{\epsilon, p}(u, v) .
$$

Since Corollary 1.1 holds for the operator $L_{\epsilon, Y}$, it follows that for every $p_{1}<p$,

$$
\left|\epsilon^{j} X_{i_{1}} \cdots X_{i_{p_{1}}} F_{\epsilon}(u, v)\right| \leq C_{p_{1}}, \quad i_{1}, \ldots, i_{p_{1}} \in\{1, \ldots, n\},
$$

where $j$ denotes the number of indices $i \in\{m+1 \ldots, n\}$, and $C_{p_{1}}$ is independent of $\epsilon$.

Since $K_{\epsilon, p}$ is a kernel satisfying the thesis of Theorem 1.1 with constants independent of $\epsilon$, the thesis immediately follows.

Proof of Corollary 1.1 It immediately follows form Theorem 1.1 and standard properties of singular integrals.

\section{REFERENCES}

[1] L. Ambrosio, S. Rigot, Optimal Mass Transportation in the Heisenberg Group, to appear.

[2] F. Antonelli, E. Barucci, M. E. Mancino, A Comparison result for FBSDE with Applications to Decisions Theory, Math. Methods Oper. Res. 54, 3, (2001) 407-423.

[3] G. Citti, E. Lanconelli, A. Montanari, Smoothness of Lipschitz-continuous graphs with nonvanishing Levi curvature, Acta Math. 188, No.1, (2002), 87-128.

[4] G. Citti, A. Pascucci, S. Polidoro, Regularity properties of viscosity solutions of a nonHörmander degenerate equation, J. Math. Pures Appl. 80, No.9, (2001), 901-918.

[5] M. Christ, A. Nagel, E.L. Stein, S. Wainger, Singular and maximal Radon transforms: Analysis and geometry, Ann. Math. (2) 150, No.2, (1999), 489-577.

[6] A. Bonfiglioli, E. Lanconelli, F. Uguzzoni, Uniform Gaussian Estimates of the fundamental solutions for heat operators on Carnot Groups, Advances in Differential equations 7, 10, (2002), 1153-1192.

[7] M. Bramanti, L. Brandolini, $L^{p}$ estimates for nonvariational hypoelliptic operators with VMO coefficients, Trans. Am. Math. Soc. 352, No.2, (2000), 781-822.

[8] G.B. Folland, Subelliptic estimates and function spaces on nilpotent Lie groups, Ark. Mat. 13, (1975), 161-207.

[9] G.B. Folland, On the Rothschild-Stein lifting theorem, Commun. Partial Differ. Equations 2, (1977), 165-191.

[10] G.B. Folland, E.M. Stein, Estimates for the $\bar{\partial}_{b}$ Complex and Analysis on the Heisenberg Group, Comm. Pure Appl. Math. 20, (1974), 429-522.

[11] B. Gaveau, Principe de moindre action, propagation de la chaleur et estimees sous elliptiques sur certains groupes nilpotents, Acta Math. 139, (1977), 95-153.

[12] R. Goodman, Lifting vector fields to nilpotent Lie groups J. Math. Pures Appl., IX. Sr. 57, (1978), $77-85$.

[13] M. Gromov, Carnot-Carathodory spaces seen from within Bellache, Andr (ed.) et al., SubRiemannian geometry. Basel: Birkhuser. Prog. Math. 144, (1996), 79-323.

[14] H. Hörmander, Hypoelliptic second-order differential equations, Acta Math. 119, (1967), 147171.

[15] H. Hörmander, A. Melin, Free systems of vector fields, Ark. Mat. 16 (1978), 83-88.

[16] D. Jerison, A. Sánchez-Calle,Subelliptic, second order differential operators, Lect. Notes Math. 1277, (1987), 46-77.

[17] N.V. Krylov,Hölder continuity and $L_{p}$ estimates for elliptic equations under general Hörmander's condition, Top. Meth. in Nonlinear Anal. 8 (1997), 249-258.

[18] A. Nagel, E.M. Stein, S. Wainger, Balls and metrics defined by vector fields I: Basic properties, Acta Math. 155, (1985), 103-147.

[19] L. Rothschild, E.M. Stein, Hypoelliptic differential operators and nihilpotent Lie groups, Acta Math. 137, (1977), 247-320.

[20] A. Sánchez-Calle, Fundamental solutions and geometry of the sum of squares of vector fields, Invent. Math. 78, (1984), 143-160. 
[21] A. Sánchez-Calle, $L^{p}$ estimates for degenerate elliptic equations Rev. Mat. Iberoam. 4, No.1, (1988), 177-185.

[22] D. Jerison, A. Sánchez-Calle, Estimates for the heat kernel for a sum of squares of vector fields, Indiana Univ. Math. J. 35, (1986), 835-854.

[23] C.L. Fefferman, A. Sánchez-Calle, Fundamental solutions for second order subelliptic operators, Ann. Math. (2) 124, (1986), 247-272.

[24] Z. Slodkowski, G. Tomassini, Weak solutions for the Levi equation and Envelope of Holomorphy, J. Funct. Anal. 101, no. 4, (1991), 392-407.

[25] E.M. Stein, Harmonic Analysis, Princeton University Press, (1993).

[26] V.S. Varadarajan, Lie groups, Lie algebras, and their representations, Graduate Texts in Mathematics. 102, New York, Springer (1984). 